\title{
Vascular anatomy defines sites of indomethacin induced jejunal ulceration along the mesenteric margin
}

\author{
A Anthony, R E Pounder, A P Dhillon, A J Wakefield
}

\begin{abstract}
Background-Indomethacin induces ulceration in the rat jejunum with sparing of the ileum. The ulcers localise between vasa recta along the mesenteric margin of the bowel, observations that have not been fully explained.

Aim-To examine the relationship between the localisation of experimental ulcers and the vascular anatomy of the rat small intestine.

Methods-The normal vascular anatomy of the rat jejunum and ileum was studied and compared using arterial carbon ink perfusion. The anatomical localisation of early and advanced lesions induced by indomethacin was examined with particular reference to the vasculature. Mucosal injury induced by feeding vessel ligation for 24 hours or brief ischaemiareperfusion injury was examined. The existence of anatomically sensitive sites to indomethacin was tested in a two dose study.

Results-In the rat jejunum, poorly vascularised sites along the mesenteric margin were highly susceptible to indomethacin induced injury, such sites being absent from the ileum. Villous contraction was a feature of both early indomethacin injury and ischaemia-reperfusion injury in the rat jejunum. Twenty four hour ligation of jejunal vasa brevia selectively induced ischaemic injury along the mesenteric margin. Two doses of indomethacin to rats did not induce greater injury than a single dose.
\end{abstract}

Conclusions-Results support the hypothesis that the rat jejunum possesses vascularly compromised sites along the mesenteric margin that are susceptible to indomethacin induced injury. Indomethacin may cause ischaemia-reperfusion injury selectively at these sites. (Gut 1997; 41: 763-770)

Keywords: indomethacin; blood supply; small intestine; ulcers; rat; vascular anatomy; ischaemia

Jejunal ulceration induced by a single oral dose of the non-steroidal anti-inflammatory drug (NSAID) indomethacin to rats is one of several chemically induced experimental models of inflammatory bowel disease. ${ }^{1}$ It has been noted that a most interesting and consistent feature of these NSAID induced experimental lesions is their propensity to occur along the mesenteric margin of the jejunum ${ }^{2-6}$ and more specifically, at sites supplied by short feeding vessels (vasa brevia, VB) located between the long feeding vessels (vasa recta, VR). ${ }^{3}$ It is of interest that in Crohn's disease small intestinal ulcers also tend to arise along the mesenteric margin where blood vessels pass through the muscularis propria, an acknowledged, but largely ignored observation. ${ }^{7}$

Early anatomical studies of the human gastrointestinal tract identified the existence of long (VR) and short (VB) feeding vessels that supplied the antimesenteric and mesenteric margins of the bowel wall respectively. ${ }^{8}{ }^{9}$ While the mesenteric margin is known to be preferentially ulcerated in the indomethacin/rat model this predilection still lacks an explanation.

In this paper we have compared the normal vascular anatomy of the rat jejunum with the ileum (sensitive and resistant sites of indomethacin induced ulceration respectively) and examined the anatomical relationship between the localisation of lesions and the vascular anatomy. We also tested the hypothesis that the predilection for such lesions to arise at anatomically susceptible sites is explained by the existence of critically perfused areas in the bowel wall which would be more prone to ischaemic injury.

\section{Materials and Methods}

MATERIALS

Male Sprague-Dawley rats (240-270 g) were kept under standard laboratory conditions and had free access to food at all times. Indomethacin was purchased from the Sigma Chemical Company (St Louis, Missouri, USA) and black carbon ink from Pelikan (Hanover, Germany). Experiments were performed under a project licence approved by the Home Office (UK).

\section{METHODS}

Vascular anatomy of the normal rat small intestine

After terminal anaesthesia (2\% halothane and fentanyl $0.15 \mathrm{mg} / \mathrm{kg}$ intraperitoneally) rats underwent midline laparotomy. Heparin (100 U) was injected into the inferior vena cava and the rats were killed by cardiac exanguination. The gastrointestinal tract was then arterially perfused with carbon ink for visualisation of the vascular anatomy. Briefly, the abdominal aorta was cannulated with 5 gauge (French) polyethylene intravenous tubing and the portal vein ruptured to permit perfusate outflow. The aorta was perfused with heparin-saline followed by carbon ink until the entire bowel had turned black. 


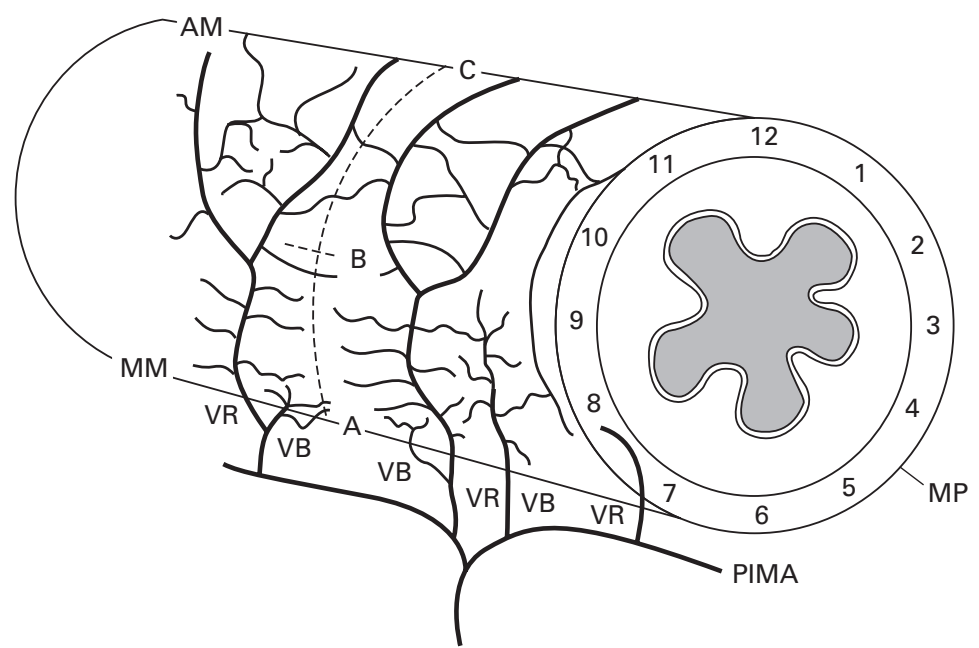

Figure 1: Schematic diagram of the rat small intestine indicating the arterial blood supply. Long (VR) and short (VB) feeding vessels arising from the peri-intestinal marginal artery (PIMA) supply the antimesenteric (AM) and mesenteric margins $(M M)$ respectively. The cross section of the bowel wall is considered to represent a clock face where the antimesenteric and mesenteric margins are located at 12 and 6 o'clock respectively.

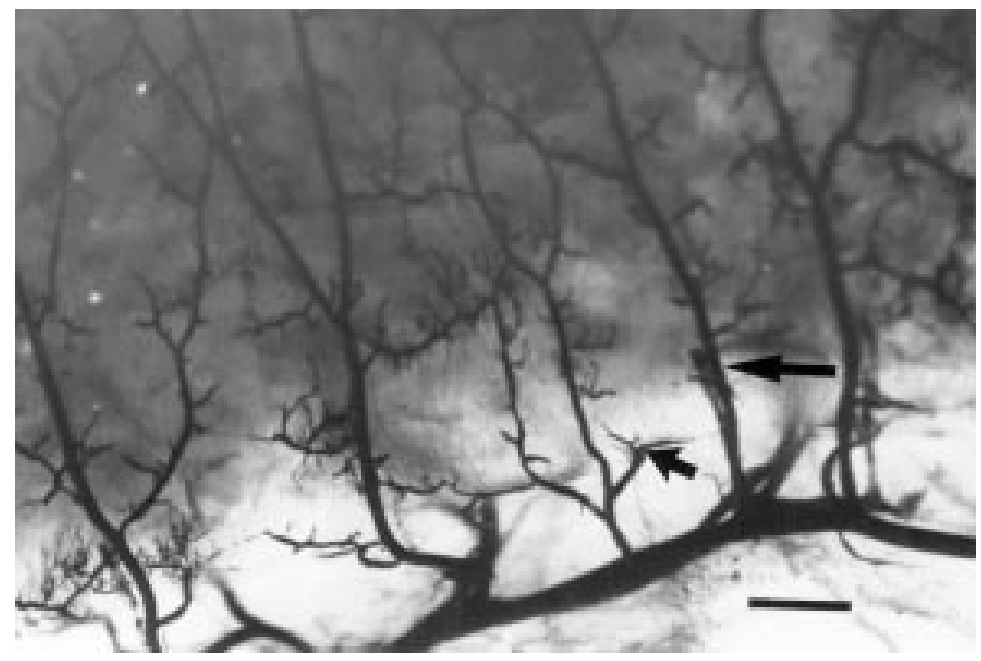

Figure 2: The mesenteric margin of an unopened loop of normal rat jejunum that has been cleared to reveal the carbon filled vasculature. Only the arterial system was filled with carbon ink. Arising from the large extramural peri-intestinal marginal artery are VR (large arrow) and the smaller VB (small arrow). Notice that the sites of penetration of the VB are closer to the mesenteric margin than the sites of VR penetration. The areas of the bowel supplied by the VB correspond to sites of indomethacin ulceration. Bar $=0.2 \mathrm{~cm}$.

Segments of mid-jejunum $(20 \mathrm{~cm})$ and terminal ileum $(10 \mathrm{~cm})$ were removed and divided into two, one half being opened longitudinally along the mesenteric margin and the other opened along the antimesenteric margin. The tissues were pinned onto cork and immersion fixed overnight. The tissues were then dehydrated in absolute ethanol and cleared to transparency in methylsalicylate using the method of Spalteholtz. ${ }^{10}$ Small segments (2 $\mathrm{cm}$ ) of jejunum and ileum were left unopened for the examination of intact tissue. The vascular anatomy was examined by dissection microscopy, focusing primarily on the feeding vessels of the bowel wall and the submucosal plexus. Distances ( $\mathrm{mm}$ ) between feeding vessels were measured using a ruler. It was possible to trim away small sections of cleared tissue from the main specimen for direct visualisation under a light microscope by securing the piece with a glass cover slip. A calibrated point counting eyepiece graticule ( $25 \mu \mathrm{m}$ intervals) was used to make vascular measurements.

To quantify the vascularity of the mesenteric and antimesenteric halves of the rat jejunum and ileum, segments of cleared tissues from each rat were examined by light microscopy as described above. A line (AC) between two consecutive VR, not separated by a VB, passing from the mesenteric margin to the antimesenteric margin was visualised using the eyepiece graduated scale (fig 1). The line was bisected into mesenteric (AB) and antimesenteric halves (BC). All arteries transecting the line in each half were scored 1,2 , or 3 according to outer diameter (score $1=25-50$ $\mu \mathrm{m}$; score $2=50-75 \mu \mathrm{m}$; score $3=75-100$ $\mu \mathrm{m})$. A total score for each half was determined by summating the scores. For each rat, five consecutive lines were assessed in this way and the mean score was calculated for the mesenteric and antimesenteric halves. The mean (SEM) score in each half was determined for the group of rats $(n=5)$.

\section{Indomethacin induced jejunal ulceration}

Two groups of rats $(n=5)$ received an oral dose of indomethacin $10 \mathrm{mg} / \mathrm{kg}$ as a sodium bicarbonate $1.5 \%$ aqueous solution.

In one group the rats were terminally anaesthetised four hours after indomethacin and the entire small intestine was examined externally for early lesions. After terminal cardiac exanguination, the small intestine was perfused arterially with carbon ink as described in the previous section. The jejunum (defined as the segment of small intestine beginning $10 \mathrm{~cm}$ distal to the pylorus and ending $20 \mathrm{~cm}$ from the ileocaecal valve) and terminal ileum (defined as the terminal $10 \mathrm{~cm}$ of intestine), were opened longitudinally along the antimesenteric margin, pinned onto cork, immersion fixed overnight, and cleared to transparency using the method described earlier. The position of each indomethacin induced early mucosal lesion and its relationship with the vasculature was noted by both dissection microscopic examination and light microscopy. Smaller pieces of tissue from the main specimen were placed on a glass slide and covered with a glass cover slip. Areas of normal mucosa from antimesenteric and mesenteric sites were also examined. Lesions and normal areas were examined histologically by trimming them from the main specimen, washing in chloroform overnight, and processing into paraffin wax for routine histology.

In the other group the rats were killed 24 hours later and the small intestine examined externally for lesions followed by longitudinal opening along the mesenteric margin for assessment of advanced mucosal ulceration.

\section{Ligation of vasa recta and vasa brevia}

Experiments involving extramural ligation of rat VR and VB feeding vessels were performed to establish which parts, if any, of the bowel wall would ulcerate after prolonged ( 24 hours) ischaemia. Ligation of VB for a shorter period followed by reperfusion was carried out to 


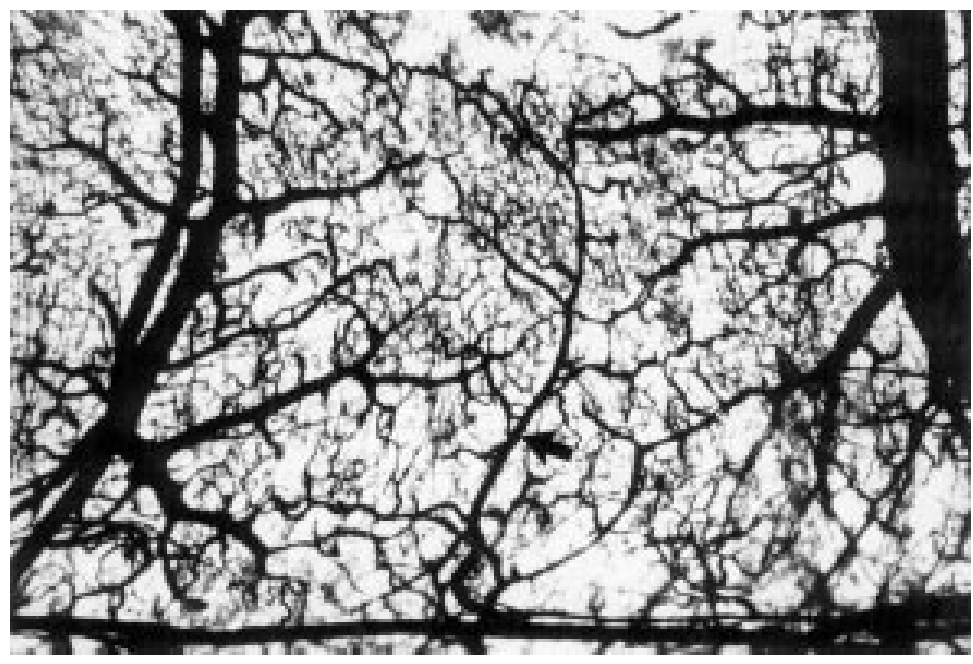

Figure 3: A section of normal rat jejunum that has been secured beneath a glass cover slip and visualised by light microscopy. $A V B$ (arrow) situated between two VR is arising directly from the peri-intestinal marginal artery that is running horizontally at the bottom of the field. The VR are seen on the far left and right. Notice the large difference in calibre between VB and VR. Original magnification $\times 10$.

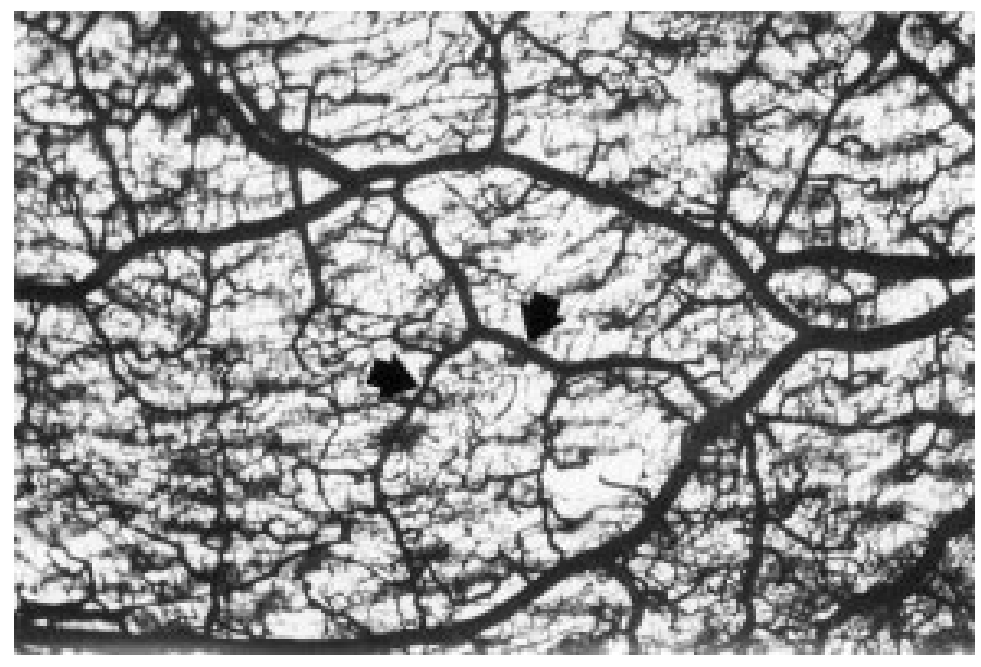

Figure 4: Submucosal vasculature within the antimesenteric aspect of the rat jejunum showing good collateral arterial connections (arrows) between large arteries. Original magnification $\times 10$

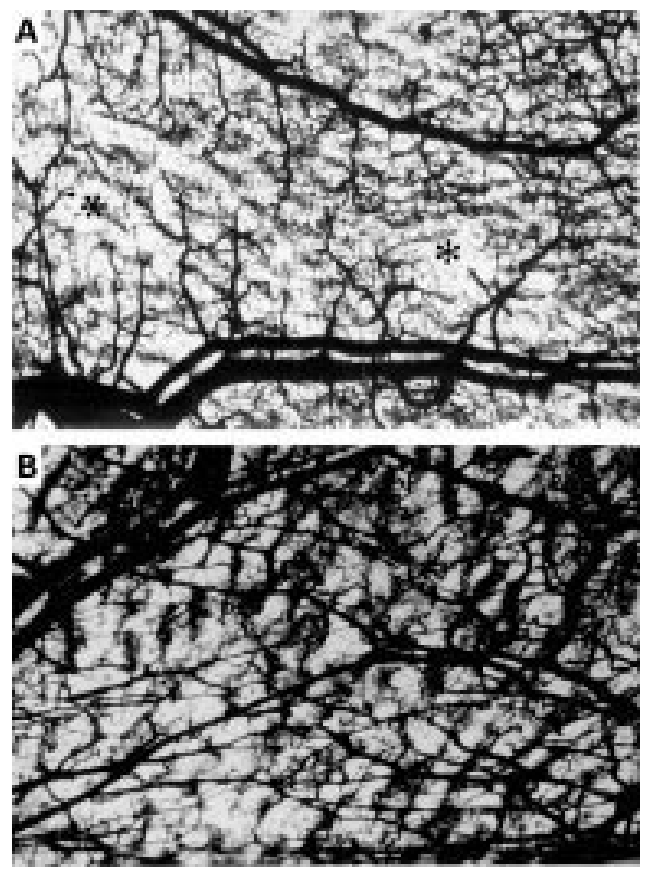

Figure 5: (A) Normal rat jejunum showing paucity of the submucosal vasculature between two VR close to the mesenteric margin. There are no interconnecting vessels between the VR where relatively avascular tissue (asterisk) is supplied by blind ending arteries. Original magnification $\times 10$. (B) Equivalent site in the rat ileum showing good arterial connection between VR along the mesenteric margin. Original magnification $\times 10$.

blood flow through a single VB was temporarily arrested by applying traction to a $6-0$ suture tied loosely around the extramural aspect for 35 minutes. Blood flow was then restored for 15 minutes and the experiment was terminated for macroscopic and histological assessment of the mucosa. The other six rats represented sham operated controls that were treated in the same way except that no traction was applied to the ligature. The segment of bowel supplied by the ligated VB (about $0.3 \mathrm{~mm}$ either side of the sutured vessel) was fixed in formalin and trimmed into four segments for histology. Villous height was assessed by measuring the crypt height:villous height ratio using a calibrated eyepiece graticule. The ratio was assessed in four villi situated within the mucosa supplied by the ligated vessel and four villi within the antimesenteric margin. The ratio was also measured in equivalent regions of the bowel wall of sham operated rats. The mean ratio for each rat was assessed at each site and the results expressed as mean (SEM) for the group $(n=6)$.

\section{Susceptibility of anatomically defined jejunal sites} to indomethacin

Three groups of rats $(n=5)$ received oral indomethacin $10 \mathrm{mg} / \mathrm{kg}$. Two of these groups were terminally anaesthetised with $3-5 \%$ halothane four and 24 hours later. The small intestine was arterial perfusion fixed, followed by histological assessment of a $20 \mathrm{~cm}$ segment of the mid-jejunum (about 80 sections) as previously described for early (four hours) and late (24 hours) histological damage. ${ }^{3}$ The third group received a second dose of oral indomethacin treated and examined in the same way.

Twelve other rats were anaesthetised and laparotomised as above. In six of these, the 
$10 \mathrm{mg} / \mathrm{kg} 24$ hours after the first followed by histological assessment four hours later. The aim of administering two doses of indometh-

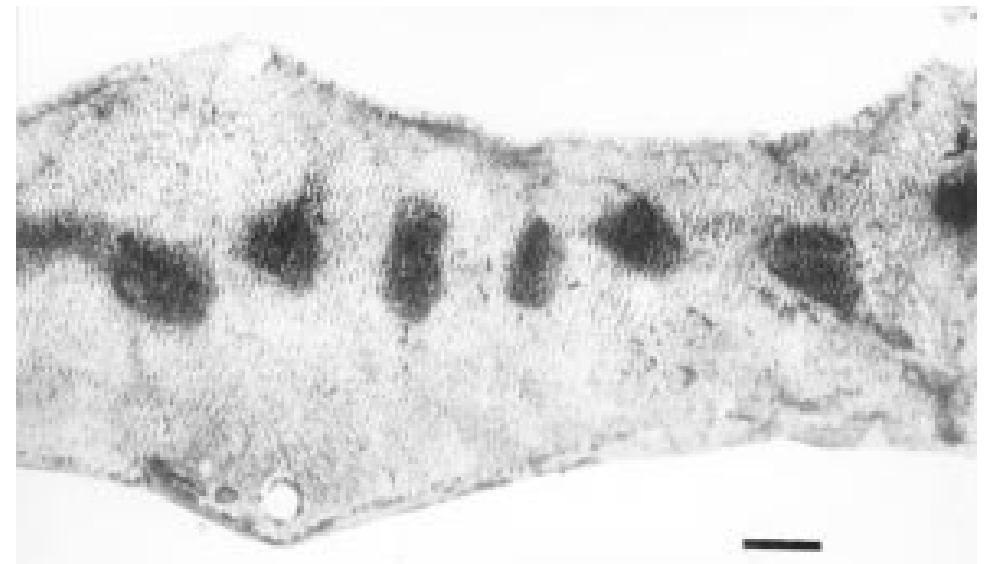

Figure 6: An opened segment of rat jejunum showing discrete, black, indomethacin induced lesions localised along the mesenteric margin. The bowel was perfused arterially with carbon ink, fixed in formalin, and dehydrated in ethanol. Bar $=0.2 \mathrm{~cm}$.

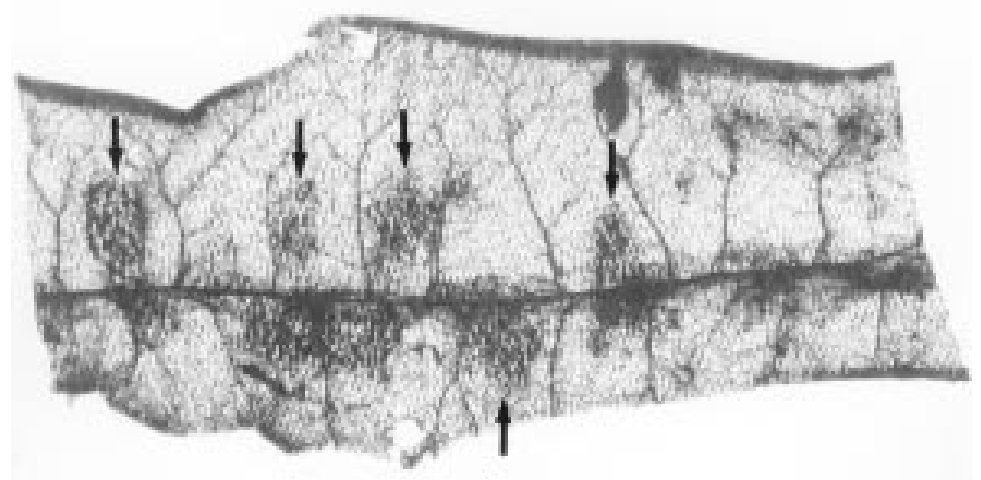

Figure 7: An opened segment of arterially perfused and cleared rat jejunum showing discrete, black, indomethacin induced lesions localised along the mesenteric margin (arrows). Most lesions are located between VR. Bar $=0.2 \mathrm{~cm}$.

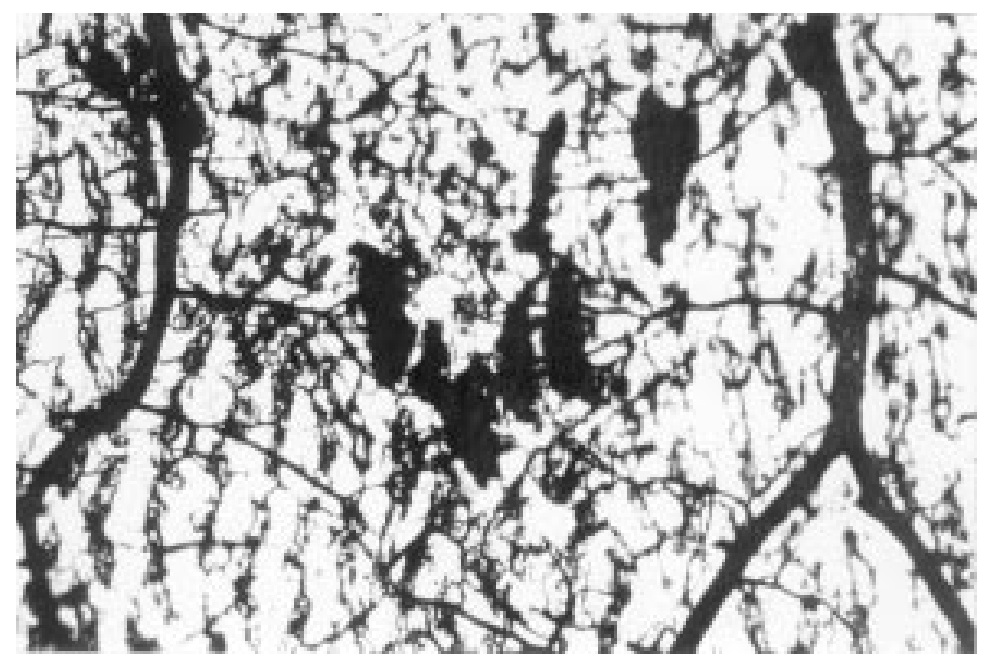

Figure 8: Light photomicrograph of cleared jejunal tissue secured between a glass cover slip and histological slide. An indomethacin induced lesion (four hours after dosing) composed of about seven abnormal villi clustered between two VR can be seen. The lesion is sited at the interface of end arteries arising from the VR. Original magnification $\times 40$. acin was to determine whether the jejunum possesses anatomically defined areas susceptible to indomethacin injury. If such areas exist, the first dose would preferentially identify these sites, and a second dose should theoretically not produce further lesions. This experiment relies on the ability to differentiate histologically between late ulcerative lesions (focal but complete mucosal loss) arising from the first dose and early preulcerative changes (villous shortening, epithelial and microvascular injury, and superficial villous necrosis) attributed to the second dose.

\section{STATISTICS}

The data were analysed using Wilcoxon rank sum test. An associated probability of $5 \%$ or less was considered significant.

\section{Results}

VASCULAR ANATOMY OF THE RAT JEJUNUM

The arterial blood supply of the rat jejunum and terminal ileum was identified as arising from the mesenteric branch of the abdominal aorta. Within the mesentery the mesenteric artery divided into about 20 terminal branches that formed a continuous peri-intestinal marginal artery running parallel, and close, to the mesenteric margin of the bowel wall. Arising from the marginal vessel were two types of feeding arteries that supplied the bowel wall and were distinguished by their length, outer diameter, and point of insertion into the bowel wall. If the cross section of the bowel wall is considered to represent a clock face, with the mesenteric and antimesenteric margins representing 6 and 12 o'clock respectively, the first type of vessel consisted of VR (diameter $200-250 \mu \mathrm{m}$ ) that branched directly from the peri-intestinal marginal artery and alternately pierced the bowel wall at approximately 5 and 7 o'clock to supply the anterior and posterior walls (figs 1 and 2). Usually accompanied by a vein, they entered the muscularis obliquely, passing through the latter for about $0.2-0.3 \mathrm{~cm}$ and bifurcated within the submucosa at 3 and 9 o'clock. The two branches arising from this bifurcation passed over to the antimesenteric side where they united with equivalent branches from the opposite side of the bowel wall, forming the submucosal collateral plexus (fig 1).

The second type of feeding vessel was shorter and of lower calibre (VB diameter $100-200 \mu \mathrm{m}$ ) arising from either the marginal artery directly or from the extramural aspect of VR, the latter type of VB being much shorter and of a lower calibre than the former (figs 2 and 3). VB entered the bowel wall at 6 o'clock, the point of entry sited between the point of entry of consecutive VR. A significant difference between the jejunum and ileum was the greater distance between consecutive jejunal VR on the same side of the bowel wall compared with the equivalent ileal distance $(V R-V R$ distance jejunum $=3.1(0.2) \mathrm{mm}$ versus ileum $=2.0(0.04) \mathrm{mm} ; \mathrm{p}<0.05)$. This difference made the ileum appear much more vascular than the jejunum and intervening jejunal VB supplied a larger area of bowel wall along the mesenteric margin. 


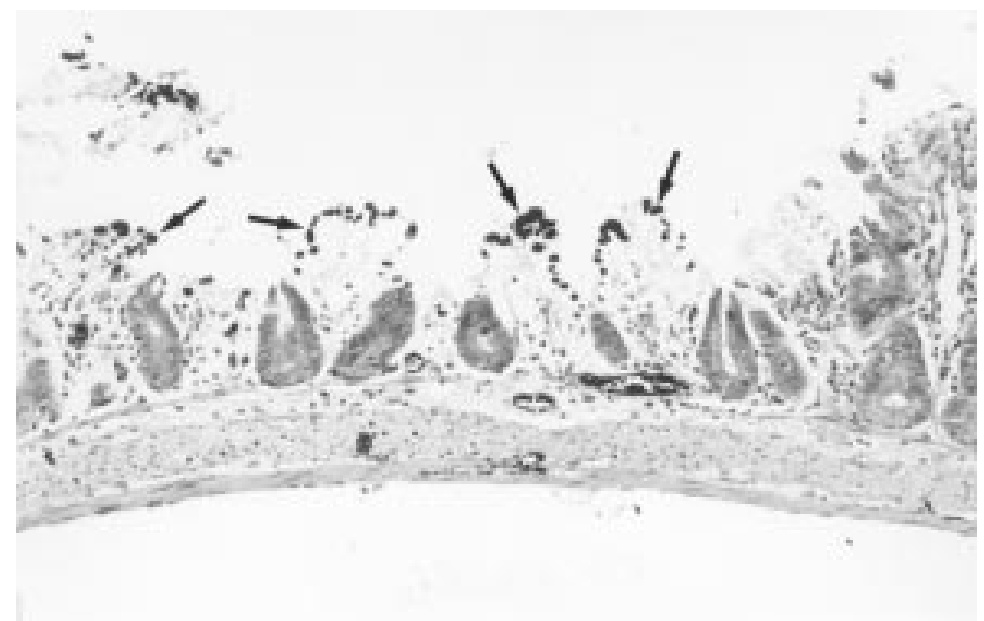

Figure 9: Photomicrograph of a mesenteric jejunal lesion showing severely contracted and degenerate villi (four hours after indomethacin). The villous capillaries are ectatic and filled with carbon ink (arrows). Haematoxylin and eosin. Original magnification $\times 100$. were apparent (fig 5B). The vascularity score of the jejunal mesenteric margin was significantly lower than that of the antimesenteric side (jejunal mesenteric score $=3.9(0.4)$ versus antimesenteric $=8.6(0.6) ; \mathrm{p}<0.05)$. There was no such vascular difference in the ileum at these sites (ileal mesenteric score $=6.5(0.6)$ versus antimesenteric $=6.6(0.2))$.

Small arterioles branched from the submucosal plexus to supply the muscularis propria, pericryptal areas, and individual villi. Apart from the main peri-intestinal marginal artery there were no intercommunicating vessels between adjacent VR or VB outside the bowel wall.

INDOMETHACIN INDUCED JEJUNAL LESIONS Indomethacin, four hours after dosing, induced multiple lesions in the mid-jejunum of all animals, no lesions being evident in the duodenum and terminal ileum. Externally, both the appearance and localisation of these early lesions were similar to those of the advanced lesions. After carbon ink perfusion they were easily identified as black, round, and oval shaped punched out mucosal depressions, measuring approximately $0.2-0.4 \mathrm{~cm}$ in diameter (fig 6). On examination of the cleared specimen, most lesions (95 (5)\%) were localised preferentially to the mesenteric margin between VR (fig 7). VB were noted to supply the larger areas (about 30-50 villi) of mucosa affected by indomethacin. The territory defined for smaller lesions was supplied by up to three small branches of VR, each small lesion being situated at the interface of these vessels (fig 8).

Histologically, four hour lesions showed severely contracted and superficially necrotic villi with ectatic surface capillaries filled with ink. Villi within the central core of the lesion were the most severely damaged (fig 9), peripheral villi showing milder changes that included villous contraction, epithelial loss, and superficial capillary distortion with little necrosis. The mucosa of both the antimesenteric margin and mesenteric margin between lesions showed mild villous shortening and superficial capillary distortion, the severity of which increased the nearer the mucosa to lesions.

Indomethacin, 24 hours after dosing, induced visible external lesions (brown circular and oval shaped spots) confined to the jejunum, most of which $(97.4(0.4) \%)$ were located along the mesenteric margin between VR. Each of these lesions corresponded to deep oval shaped mucosal ulcers arranged along the mesenteric margin, and separated by a segment of normal mucosa. Histological examination showed focal mucosal loss with neutrophil infiltration of the ulcer base and muscularis propria.

LIGATION OF VASA RECTA AND VASA BREVIA

Twenty four hours after ligation of the feeding vessels, four of the six rats developed small single mucosal ulcers $(0.5 \mathrm{~mm})$ directly overlying the ligated jejunal VB. These small lesions were situated along the mesenteric margin with 

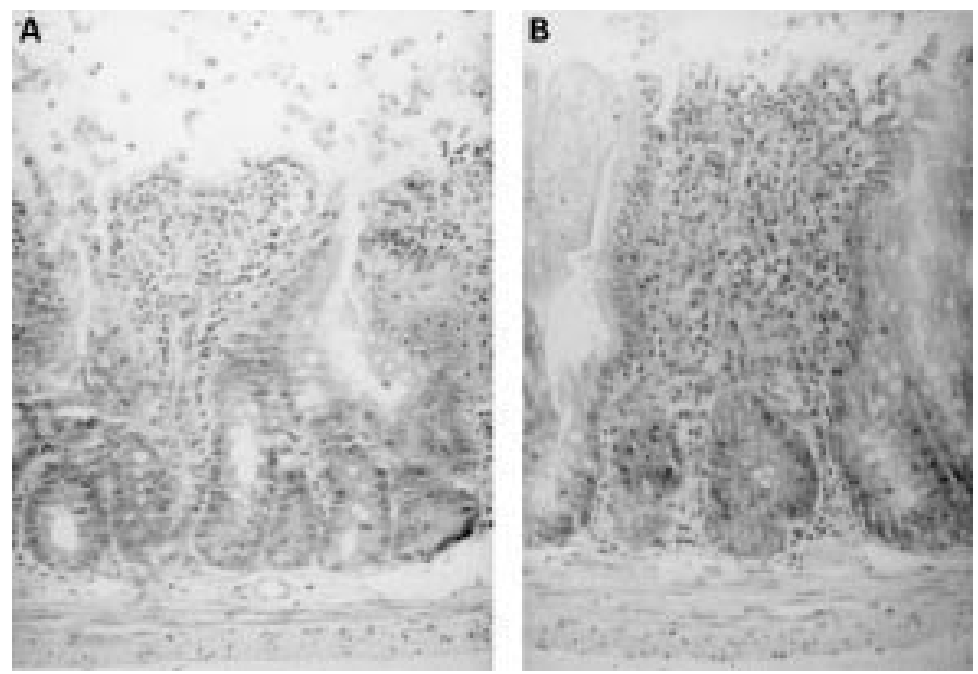

Figure 11: Photomicrographs of mucosa from a rat in which a single VB was ligated for 35 minutes followed by 15 minutes of reperfusion. The villus in $A$ was localised to an area of the mucosa supplied by the occluded-reperfused vessel along the mesenteric margin and shows severe contraction, while the villus of normal appearance in $B$ was localised to mucosa along the antimesenteric margin. Original magnification $\times 100$.

sparing of the antimesenteric margin. No lesions were noted in the ileum or in the segment of jejunum in which the VR were ligated. Histologically, all lesions showed both focal mucosal necrosis and neutrophil infiltration at the mesenteric margin (fig 10), microscopic appearances that were quite similar to those noted 24 hours after oral indomethacin. No lesions were identified in the other two rats. None of the sham operated rats developed lesions.

Sham ligation had no significant morphological effect on the jejunal mucosa but ligation-reperfusion of a single jejunal VB caused significant focal villous shortening within the adjacent mucosa along the mesenteric margin (fig 11; mean (SEM) villous height:height ratio in VB ligated rats, mesenteric villi $=0.87(0.05)$ versus antimesenteric $=0.67(0.06), \mathrm{p}<0.05 ;$ in non-ligated sham rats, mesenteric villi $=0.65(0.07)$ versus antimesenteric villi $=0.64(0.05))$. The villous shortening was confined to the area of the bowel wall considered to be supplied by the ligated vessel while the surrounding mucosa serving as internal control was normal.

\section{SUSCEPTIBILITY OF ANATOMICALLY DEFINED}

JEJUNAL SITES TO INDOMETHACIN

A single dose of oral indomethacin $10 \mathrm{mg} / \mathrm{kg}$ to rats induced histological changes in approximately half of the jejunal sections studied, four

TABLE 1 Evaluation of indomethacin susceptible sites in the rat duodenum

\begin{tabular}{llll}
\hline \multirow{4}{*}{ Treatment } & \multicolumn{2}{l}{ Percentage of histological section showing: } \\
\cline { 2 - 4 } & Early changes & Late changes & No abmormality \\
\hline $4 \mathrm{~h}(1$ dose $)$ & $58.8(10.4)$ & 0 & $41.4(11.0)$ \\
$24 \mathrm{~h}(1$ dose $)$ & 0 & $47.4(9.7)$ & $52.6(9.7)$ \\
$24+4 \mathrm{~h}(2$ doses $)$ & 0 & $38.4(7.3)$ & $61.2(7.3)$ \\
\hline
\end{tabular}

Two groups of rats received a single oral dose of indomethacin $10 \mathrm{mg} / \mathrm{kg}$; the jejunum was assessed histologically four and 24 hours later for early and late changes. A third group received a second dose of indomethacin 24 hours after the first dose and the jejunum was assessed for histological changes four hours later. Early $(4 \mathrm{~h})$ histological changes were defined as focal villous shortening, superficial villous necrosis and neutrophil infiltration. ${ }^{3}$ Late $(24 \mathrm{~h})$ histological changes were defined as focal full thickness mucosal necrosis and neutrophil infiltration. and 24 hours after dosing; the remaining half from each group were normal (table 1). Early changes (four hours) consisted of villous shortening, superficial villous necrosis, and neutrophil infiltration. Late changes consisted of full thickness mucosal necrosis and neutrophil infiltration. No early histological changes were seen in the rats receiving two doses of indomethacin; late changes were observed in $38.4 \%$ of the sections and $61.2 \%$ were normal. There was no significant difference in the number of late lesions in rats receiving either one or two doses of indomethacin.

\section{Discussion}

In this study of the vascular anatomy of the rat small intestine, we have identified a site along the mesenteric margin of the jejunum that is susceptible to both early and late phases of indomethacin induced injury. The vascularity of this site was significantly lower than the equivalent site along the antimesenteric margin, giving the clear impression that the arterial blood supply of the mesenteric margin mucosa might become preferentially compromised during an ischaemic insult to the small intestine. An equivalent avascular site was not identified in the rat terminal ileum, a section of the small bowel that is indomethacin resistant.

Mucosal ulceration only occurred at the jejunal mesenteric margin following ligation of jejunal VB while ligation of jejunal VR and ileal VB and VR failed to induce ischaemic injury. This is strong evidence that the mesenteric marginal site supplied by VB might represent a critically perfused part of the jejunum that is not served by a collateral blood supply and is therefore susceptible to ischaemia. Evaluation of the normal vascular anatomy would suggest that jejunal VB are effectively end arteries.

The observation that a second dose of indomethacin to rats failed to cause additional injury to the jejunum may be interpreted as exhaustion of anatomically susceptible sites by the first dose, leaving no sites available for the second dose to damage.

The observation by others that parenteral, as well as oral, dosing with indomethacin is able to induce jejunal lesions in the rat, ${ }^{11}$ and the preference for lesions to arise at anatomically defined sites in this study, suggest that a systemic effect of the drug is likely to play an important role in the pathogenesis of lesions. However, exposure of the jejunal mucosa to both luminal and systemic indomethacin is necessary for the early reduction in villous blood flow as demonstrated in ex vivo videomicroscopic experiments in rats. ${ }^{12}$ Luminal bacteria, food, and bile acids are believed to play important secondary roles in the genesis of lesions in this animal model. ${ }^{211}{ }^{13}$ Bile duct ligation of rats prior to administration of either oral or parenteral indomethacin prevents jejunal ulceration. ${ }^{11}$ Indomethacin is known to undergo extensive enterohepatic cycling; the protective effect of bile duct ligation would therefore imply that systemic levels of indomethacin alone are insufficient to cause inflammation and necrosis of the mucosa. ${ }^{13}$ 
Inhibition of prostaglandin synthesis by NSAIDs was thought to play a role in gastrointestinal injury caused by this class of drug. However, Whittle observed a dissociation of the appearance of the damaging effects of indomethacin and prostaglandin synthesis that does not support such a role for prostaglandins. ${ }^{14}$ Furthermore, not all NSAIDs that inhibit small intestinal prostaglandin synthesis cause ulceration at this site. ${ }^{15}$

Neutrophil recruitment by endothelial cell adhesion has also been proposed to play a primary role in NSAID damage to the gut ${ }^{16}$ but recent studies have questioned the idea that NSAID gastrointestinal injury is neutrophil dependent. $^{3}{ }^{1718}$ It is certainly difficult to explain the distribution of intestinal lesions on the basis of the studies claiming prostaglandin inhibition and neutrophil recruitment to be primary pathogenic mechanisms operating in this situation.

Precisely how indomethacin might influence blood flow to the small intestine is not known, but intravenous administration of indomethacin has resulted in a rapid splanchnic vasoconstriction in anaesthetised dogs. ${ }^{19}$ By reducing jejunal blood flow, ulcerogenic doses of indomethacin might cause a generalised ischaemia of the small intestine that is sufficient to cause macroscopic damage at "watershed" sites. The VB ligation studies presented in this paper and the superior mesenteric artery ligation experiments of Boros et $a l$, also performed in the rat, ${ }^{20}$ suggest that villous shortening is a physiological response to mucosal ischaemia-reperfusion. Support for the involvement of vasoconstriction in this situation is the observation that the $\beta_{3}$ adrenoceptor agonist CL316243, known to have vasodilator activity in the gastrointestinal tract, ${ }^{21}$ is a potent inhibitor of indomethacin induced jejunal ulceration in the rat. ${ }^{22}$ Reversal of early histological villous shortening, a preulcerative phase of injury thought to result in disruption of villous blood flow, was noted to be associated with CL316243 protection in that study. ${ }^{22} \mathrm{Ex}$ vivo videomicroscopy has shown slowing, and finally stopping of villous blood flow, 20 minutes after combined parenteral and luminal administration of indomethacin to the jejunal mucosa of anaesthetised rats, changes that could only be recorded in villi located between VR. ${ }^{12}$

An alternative explanation for the focal nature of the lesions might involve contraction of the muscularis propria. Contraction of the muscularis propria has been implicated by Takeuchi et $a l^{23}$ and Mersereau and Hinchey ${ }^{24}$ to play a role in NSAID induced gastric ulceration in the rat. They noted that lesion formation correlated with an increase in gastric smooth muscle contraction and motility; both lesion formation and muscular contraction were prevented by the spasmolytic muscarinic antagonist, atropine. Whether muscular contraction and motility are factors in the pathogenesis of NSAID injury to the small intestine has yet to be determined, but $\beta_{3}$ adrenoceptor agonists, known to have spasmolytic activity in the rat small intestine, ${ }^{25}$ and atropine, are both inhibitors of indomethacin induced jejunal ulceration in the rat. ${ }^{22} 2526$

The predilection of the mesenteric margin to form lesions specifically at a site supplied by smaller feeding vessels raises the possibility that the latter might be more susceptible than the larger vessels to muscular compression. $\mathrm{Pi}-$ asecki found that the propensity of human non-NSAID peptic ulcer disease to occur along the lesser curvature of the gastric antrum was directly related to the increased prevalence of "mucosal end arteries of extramural origin" at that site. ${ }^{27}$ The author proposed that muscular compression or vasoconstriction of these functional end arteries during periods of stess would result in mucosal ischaemia and ulceration. ${ }^{28}$ Livingston et al showed that electric field stimulated contractions of the rat stomach could reduce or stop mucosal blood flow. $^{29}$

We noted that the superficial capillaries within the degenerate villous tips were ectatic and noticeably filled with ink four hours after indomethacin. This might arise if muscular contraction was only of sufficient intensity to cause venous, but not arterial, occlusion although an alternative explanation might be that damaged capillaries are easily distendable during filling with ink.

In conclusion, we have identified a defined anatomical site along the mesenteric margin of the rat jejunum that is susceptible to both the early and late phases of indomethacin induced ulceration. The vascularity of this site is significantly lower than the equivalent area along the antimesenteric margin and ligation studies suggest that the blood supply to it does not communicate with the submucosal plexus of vessels that exists in the rest of the bowel. This site seems to represent a critically perfused "watershed" area of bowel that could be more prone to ischaemia and reperfusion injury.

1 Elson CO, Sartor RB, Tennyson GS, Riddell RH. Experimental models of inflammatory bowel disease. Gastroenterology 1995;109:1344-67.

2 Kent TH, Cardelli RM, Stamler FW. Small intestinal ulcers and intestinal flora in rats given indomethacin. Am $\mathcal{F}$ Pathol 1969;54:237-49.

3 Anthony A, Dhillon AP, Nygard G, Hudson M, Piasecki C, Strong P, et al. Early histological features of small intestinal injury induced by indomethacin. Aliment Pharmacol Ther 1993;7:29-40.

4 Anthony A, Dhillon AP, Thrasivoulou C, Pounder RE, Wakefield AJ. Pre-ulcerative villous contraction and microvascular occlusion induced by indomethacin in the rat jejunum: a detailed morphological study. Aliment Pharmacol Ther 1995;9:605-14.

5 col Ther 1995;9:605-14. Fujishima M. Preventive effect of immunosuppressive Fujishima $M$. Preventive effect of immunosuppressive

ulcers in rats. Dig Dis Sci 1994;39:787-95.
6 Wax J, Clinger WA, Varner P, Bass P, Winder CV. Relationship of the enterohepatic cycle to ulcerogenesis in the rat small bowel with flufenamic acid. Gastroenterology 1970;58: $772-80$

7 Brahme F, Lindstrom C. A comparative radiographic and pathological study of intestinal vaso-architecture in Crohn's disease and in ulcerative colitis. Gut 1970;11:92840.

8 Noer RJ. The blood vessels of the jeunum and ileum: a comparative study of man and certain laboratory animals. $A m \mathcal{F}$ Anat 1943;73:293-334.

9 Griffiths JD. Extramural and intramural blood-supply of colon. BMF 1961;1:323-6.

10 Spalteholtz H. Ruber das durchsidutigmachen von menschlichen und tierischen preparaten. S Histzol Leipzig, schlich 1914 .

11 Brodie DA, Cooke PG, Bauer BJ, Dagle GE. Indomethacininduced intestinal lesions in the rat. Toxicol Appl Pharmacol 1970;17:615-24.

12 Kelly D, Piasecki C, Anthony A, Pounder RE, Wakefield AJ. Early indomethacin lesions in rat jejunum: reduced focal 
blood flow and shortening of villi precede ulceration [abstract]. Gastroenterology 1996;110:A337.

13 Duggan DE, Hooke KF, Noll RM, Kwan KC. Enterohepatic circulation of indomethacin and its role in intestina irritation. Biochem Pharmacol 1975;25:1749-54

14 Whittle BRJ. Temporal relationship between cycloxygenase inhibition, as measured by prostaglandin biosynthesis, and the gastrointestinal damage induced by indomethacin in the rat. Gastroenterology 1981;80:94-8.

15 Reuter BK, Davies NM, Wallace JL. Non-steroidal antiinflammatory drug enteropathy in rats: role of permeability, bacteria and enterohepatic circulation. Gastroenterology 1997;112:109-17.

16 Wallace JL. Non-steroidal anti-inflammatory drug gastropathy and cytoprotection: pathogenesis and mechanisms re-examined. Scand 7 Gastroenterol 1992;27(suppl):3-8.

17 Melarange R, Gentry C, Toseland N, Smith PH, Fuller J. Neutropenia does not prevent etodolac- or indomethacinNeutropenia does not prevent etodolac- or indomethacininduced gastrointe

18 Anthony A, Sim R, Dhillon AP, Pounder RE, Wakefield AJ Gastric mucosal contraction and vascular injury induced by indomethacin precede neutrophil infiltration in the rat. Gut 1996;39:363-8.

19 Feigen LP, King LW, Ray J, Beckett W, Kadowitz PJ. Differential effects of ibuprofen and indomethacin in the regional circulation of the dog. F Pharmacol Exp Ther 1981;219:67984.

20 Boros $M$, Tackaichi S, Hatanaka K. Ischaemic timedependent microvascualr changes and reperfusion injury in the rat small intestine. F Surg Res 1995;59:311-20.

21 Kuratani K, Kodama H, Yamaguchi I. Enhancement of mucosal blood flow by beta-3-adrenergic agonists prevents indomethacin-induced antral ulceration in the rat. $\mathcal{F}$ Pharmacol Exp Ther 1994;270:559-65.

22 Anthony A, Bahl AK, Dhillon AP, Oakley IA, Piasecki C,

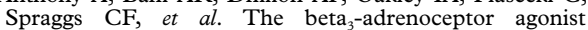
CL3162343 prevents indomethacin-induced jejunal ulceration in the rat by reversing early villous shortening. $f$ Pathol 1996;179:340-6.

23 Takeuchi K, Okada M, Ebara S, Osano H. Increased microvascular permeability and lesion formation during gastric hypermotility caused by indomethacin and 2-deoxy-Dglucose in the rat. $f$ Clin Gastroenterol 1990;12(suppl glucose in
S $76-84$

24 Mersereau WA, Hinchey EJ. Synergism between acid and gastric contractile activity in the genesis of ulceration and haemorrhage in the phenylbutazone-treated rat. Surgery 1981;90:516-22.

25 MacDonald A, Forbes IJ, Gallacher D, Heeps G, McLaughlin. Adrenoceptors mediating relaxation to catecholamines in rat isolated jejunum. Br $\mathcal{F}$ Pharmacol 1994;112:576-8.

26 Karadi O, Abdel-Salam OME, Bodis B, Moszic G. Preventive effect of atropine on indomethacin-induced gastrointestinal mucosal and vascular damage in rats. Pharmacology 1996;52:46-55.

27 Piasecki C. Blood supply to the human gastroduodenal mucosa with special reference to ulcer bearing areas. F Anat 1974;118:295-335.

28 Piasecki C. Evidence for an infarctive pathogenesis of acute and chronic gastroduodenal ulceration. F Physiol Pharmacol 1992;43:99-112.

29 Livingston EH, Howard TJ, Garrick TR, Passaro EP, Guth $\mathrm{PH}$. Strong gastric contractions cause mucosal ischaemia. Am f Physiol 1991;260:G524-30. 